\title{
Retraction of: Acupuncture Compared to Conscious Sedation for Pain Relief During In-Vitro Fertilization Oocyte Retrieval by Shu Li Cui, et al. Med Acupunct. 2020; epub: 28 Apr 2020; DOI: $10.1089 /$ acu.2020.1416
}

$\mathbf{I}$

N ApRIL 2020, Medical Acupuncture published the epub version of the article entitled, Acupuncture Compared to Conscious Sedation for Pain Relief During In-Vitro Fertilization Oocyte Retrieval by Shu Li Cui, et al. (Med Acupunct. 2020; epub: 28 Apr 2020 https://doi.org/10.1089/acu.2020.1416). Since its online publication, it has been determined that the article requires an official retraction.

After the article was published online ahead of print, it was sent to The American Academy of Medical Acupuncture (AAMA; accredited by the Accreditation Council for Continuing Medical Education (CME) and the official society of Medical Acupuncture), to be considered for qualification of CME credits. During the AAMA's review of the article, it was discovered that the authors had not provided any patient baseline pain scores, which was relevant as pain was the principle measured outcome of the study.

The AAMA asked the author for the baseline pain scores for each group, which they supplied, along with the $P$ values. Upon reviewing the baseline pain scores, it was apparent that the baseline scores for the two groups were significantly different, which was deemed as a difficult place to start a randomized controlled trial as the treatment groups are supposed to be randomized in a way so that they are equal for important variables at baseline. This difference in baseline pain scores creates difficulty in interpreting the findings in this study. The differences in the baseline readings for the two groups are an insurmountable issue, and therefore, the article is retracted.

The Editor and Publisher of Medical Acupuncture, and The American Academy of Medical Acupuncture, are committed to preserving the scientific record and the community it serves, and therefore officially retracts the article. 